\title{
SPATIAL AVERAGING APPROACH FOR DETERMINING WIND PRESSURES AND FORCES ON HIGH-RISE BUILDINGS
}

\author{
ALEKSANDER PISTOL, BARTOSZ KIESIEWICZ \& ANDRZEJ FLAGA \\ Wind Engineering Laboratory, Faculty of Civil Engineering, Cracow University of Technology, Poland
}

\begin{abstract}
The paper describes wind tunnel model tests conducted at Wind Engineering Laboratory of Cracow University of Technology, which focused on a planned high-rise building (165 m tall) located in Warsaw, Poland. The aim of the tests was to determine the wind action on the subject building's structure and claddings. A total of 383 measuring points were distributed on the external surfaces of the building. Local values of wind pressures and suctions at each measuring point were calculated on the basis of wind tunnel measurements using quasi-static extreme peak values envelope model. These values may be used to design the cladding of the building. However, in order to determine the global values of wind pressure/wind forces on the structure, a spatial averaging of these values is required. This allows taking into account the vortex scale in relation to the size of the building, that is, the fact that the peak values of wind pressure are not present simultaneously at each measuring point. Such an approach can render the results obtained from model tests closer to the reality. It provides a conservative estimation of wind pressure values without too large overestimation of the wind actions, which could result in unnecessary overdesigning of structure elements and larger material expenditure. The paper discusses in detail the method suggested by the authors and the obtained results. The results are provided in the form of 3D surface plots on the building's façades and global wind forces acting on the whole structure. The obtained results allowed the consulting engineers to adopt reliable and robust values of wind pressure acting on the structure. These values are calculated taking into account both the non-stationary, non-uniform nature of the wind and aerodynamic interference of the building's surroundings, which in large portion consists of other high-rise buildings.

Keywords: wind tunnel tests, high-rise buildings, quasi-static model, design wind action, spatial averaging.
\end{abstract}

\section{INTRODUCTION}

Providing the detailed information on environmental wind loads on structures, especially those of unorthodox shape and especially large sizes, to the designers and constructors, has always been one of the key aims of wind engineering. Various theoretical models have been developed for transition of model tests results into real life scale. They need to account for highly non-stationary wind field its large fluctuations caused by the turbulence and also for simplifying the dynamic effect into a quasi-static approach, more convenient for the designers.

The approach proposed in this paper allows for a practical transition of model tests results directly into wind loads acting on structure while accounting for the vortex scale and nonsimultaneous nature of gusts on large structures. This method is presented on an example of $165 \mathrm{~m}$ tall high-rise building, which is developed in Warsaw, Poland.

\section{EXPERIMENTAL SETUP}

\subsection{Subject of model tests}

The tests were performed for a high-rise building Skyreach (165 $\mathrm{m}$ tall) located in the inner centre of Warsaw in a densely developed surroundings [1]. The site consists of the building, 
rectangular in plan shape, and open town plaza. Fig. 1 presents the architectural visualisation of the building. In the same quarter there are other high-rise buildings, most notably Platinum Towers (twin buildings measuring $85 \mathrm{~m}$ each) and a tall complex of residential buildings reaching up to $37 \mathrm{~m}$ from the south and high-rise buildings measuring $85-100 \mathrm{~m}$ from east and west. Fig. 2 presents the satellite photo of the location, with the tested building marked in yellow.

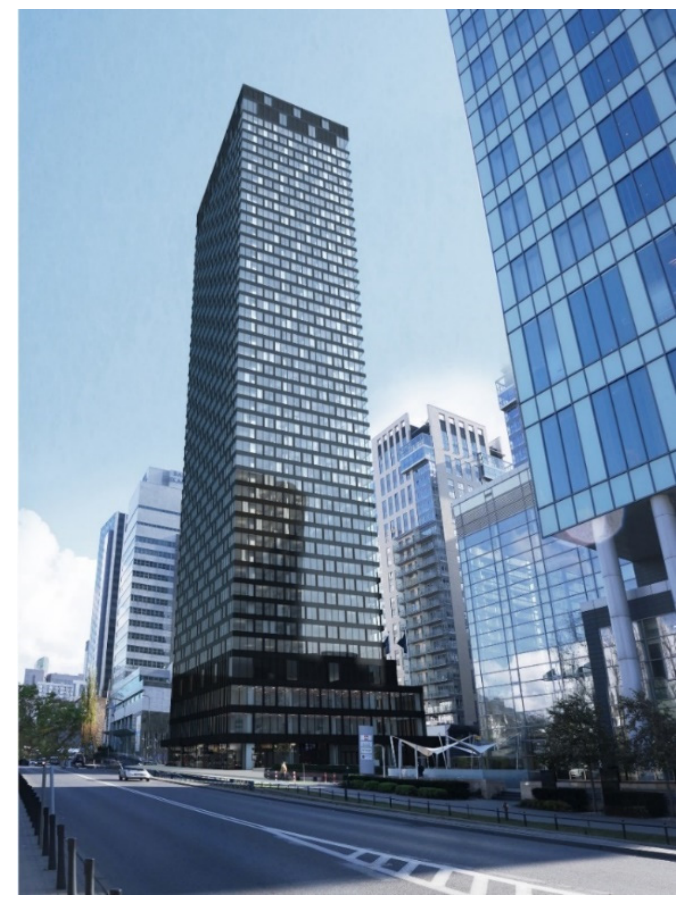

Figure 1: Computer visualisation showing the designed building and its surroundings.

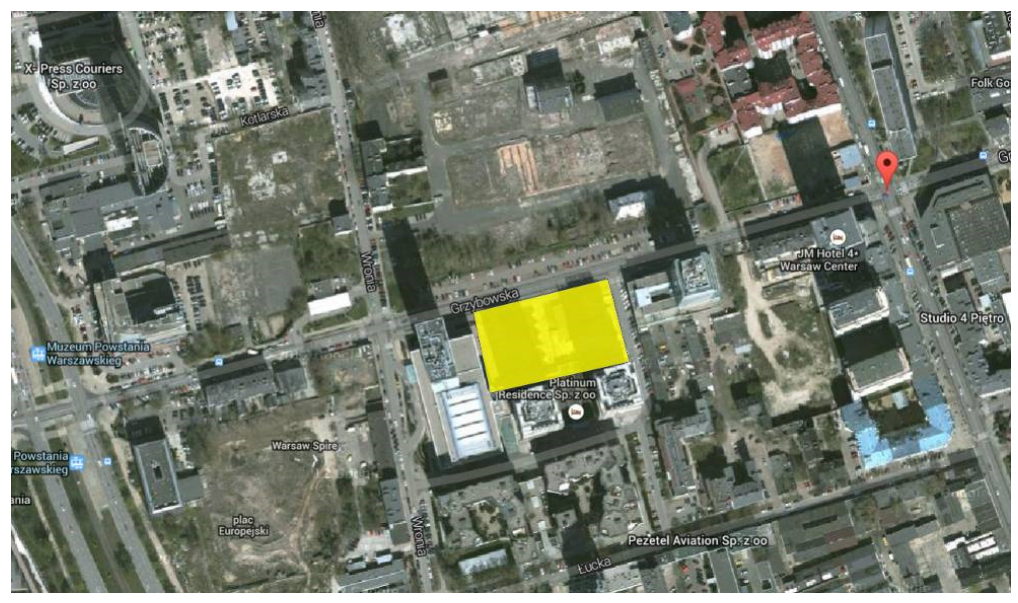

Figure 2: Satellite photo showing the building site (marked in yellow) and its surroundings. 


\subsection{Measuring apparatus}

Model tests were conducted in the boundary layer wind tunnel of Cracow University of Technology, described in detail in [2]. DTC Initum pressure scanners system was used for analogue signal data acquisition and digitalization. Scanning modules of the system are shown in Fig. 3.

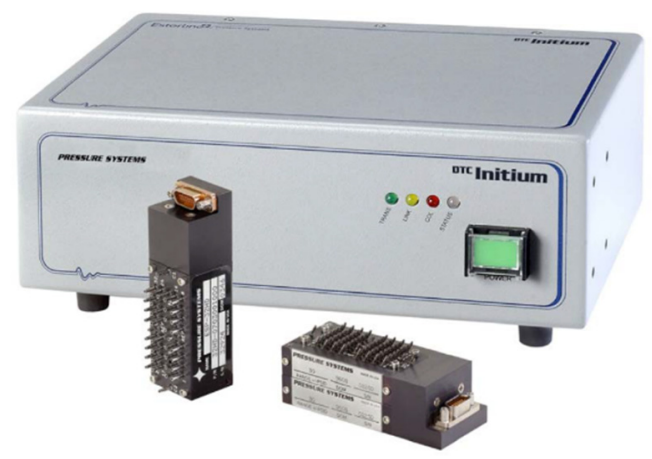

Figure 3: DTC Initum pressure scanners system.

\subsection{Model used for the tests}

Model tests were performed in the scale of 1:300. The adopted scale allowed for obtaining the most reliable results in the conditions of strong aerodynamic interference caused by the neighbouring tall buildings around the site. The model included the subject building and its nearest surroundings in the radius of $300 \mathrm{~m}$. The model was made on a rotating circular board $198 \mathrm{~cm}$ in diameter. Computer visualisation of the model is shown in Fig. 4.

The height of the subject building in the adopted scale was $57 \mathrm{~cm}$. Other high-rise buildings have been recreated on the model, some of them on the very edge of it, but their

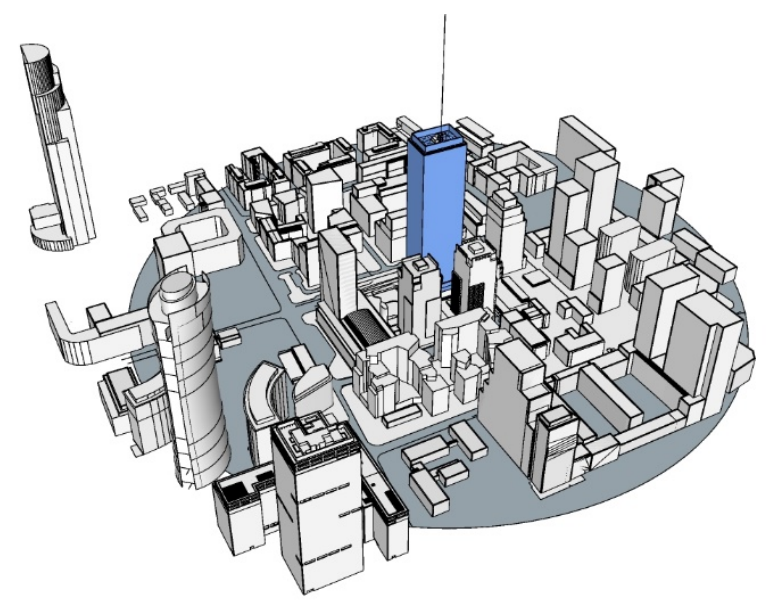

Figure 4: Computer visualisation of the model - subject building marked in blue. 
potential influence was significant enough to justify adding them. These buildings are most notably Generation Park (45 $\mathrm{cm}$ in the adopted scale), Warsaw Spire $(62 \mathrm{~cm})$, Warsaw Trade Tower $(67 \mathrm{~cm})$ and Platinum Towers $(28 \mathrm{~cm}$, two towers very close to the subject building). The completed model inside wind tunnel is shown in Fig. 5.

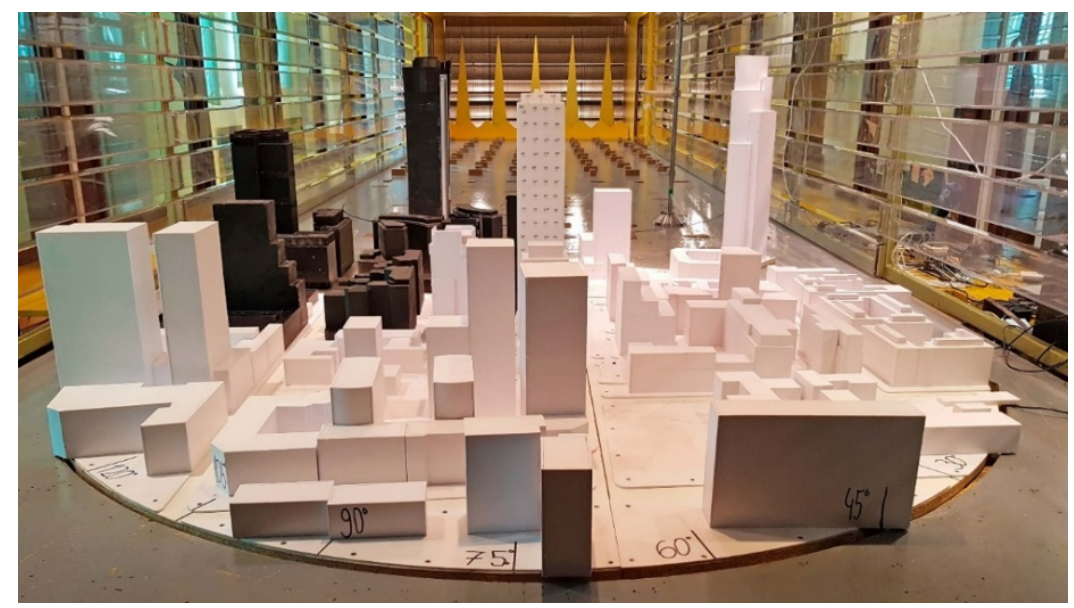

Figure 5: Completed model inside wind tunnel working section (leeward view).

\section{SCIENTIFIC METHOD}

\subsection{Theoretical basics of the proposed approach}

One of the most important models of turbulent local wind action is the quasi-stationary wind pressure model. This is a simplification of the reality which assumes that the wind action on a building under a non-stationary, turbulent wind inflow might be modelled as the inflow was stationary. More detailed theoretical considerations and model experiments in wind tunnel about this model can be found in [3].

Quasi-stationary model assumes that the building itself does not create any additional fluctuations of wind pressure, which would be close to the truth in case of large scale vortexes (larger than the size of the building). The accuracy of the discussed model strongly depends on shape and size of the building, and location of measurement point on its walls. It is completely inadequate for the calculation of wind pressures on inner walls of a building (e.g. inner surface of a cooling tower).

This approach is often used for local, partial or global aerodynamic forces and moments acting on slender, block or planar objects, but its precision is not always satisfactory. Such cases require modifications, e.g. time or spatial averaging of wind velocity fluctuations.

Wind action on structures under buffeting conditions is of dynamic nature. In cases where these objects are sufficiently rigid, the analysis may omit inertia and damping forces. This is a static approach to dynamic wind action - quasi-static models. In design practice it is convenient to use such an approach, which estimates the wind action basing on a replacement model of wind action, both on the conservative side and rationally extrapolating the formulas describing wind action. The most common models are extreme peak values envelope model and equivalent peak values model, described below. 
The first one may be based on quasi-stationary theory formulas focusing on mean values of actions. The modification here is replacing mean wind velocity $\bar{v}$ with peak wind velocity $v_{s}$ from short averaging time (e.g. 3 seconds). Such an action is fictional, replacement, usually significantly different from reality. This is for two reasons. Primarily, such an extrapolation does not have physical basis in aerodynamics. Secondarily, peak wind velocities, which result from gusts of different time and spatial scales, are not present simultaneously at all points on the building. As such, this model is conservative, on the safe side. Peak wind action calculated on its basis may be considered as an envelope of extreme wind actions.

Equivalent peak values model differs from the extreme peak values envelope model in changing peak velocity $v_{s}$ on an equivalent velocity $v_{e}$, which results from gusts in significantly large spatial scales, comprising of a large portion of the structure, for example half of a tower or half of a bridge. Therefore, the main problem is determining the minimal averaging time which would correspond to gusts of such scales. This can be done by using wind velocity frequency scales, which are used to measure frequency of wind velocity gusts at a given spatial scale.

This method is widely used in codes [4]. Quasi-static models are in general a basis of the approach proposed in Eurocodes [5].

Another modification of the basic quasi-stationary theory includes time or spatial averaging of fluctuation components of wind velocity in characteristic domains, either time or spatial. Linear operators which appear in the definitions of time or spatial averaged fluctuation components or wind directions are special cases of linear filters. Therefore, operations of time and spatial averaging are not the same, and each lead to different smoothening of the input signals.

The idea of spatial averaging in quasi-stationary theory was introduced and widely used in [6]-[8] for along-wind, across-wind and torsional wind actions on slender structures. Spatially averaged wind velocity and direction naturally appear in the formulas for wind action estimates if these formulas are derived from momentum (aerodynamic forces) and angular momentum (aerodynamic moment) principles.

The more described analysis and full formulas derivations of the models presented in the paper may be found in [9].

The approach proposed by the authors is a modified equivalent peak values model. It introduces to the analysis an intermediary step where the momentary values of wind pressure coefficients are spatially averaged for a couple of neighbouring measurement points (which form a measuring area) on a single building surface. This allows for "smoothening" the time series of wind pressure coefficients and taking into account the fact that some of the gusts act only on a very limited, local part of the façade. Moreover, a proper division of the external walls of the building for measuring areas allows for consideration of local effects on building's edges and corners, where their presence actually has a significant influence on global wind action on the structure.

\subsection{Detailed description of test procedure}

The model tests conducted in wind tunnel allowed for determination of wind pressure on façades and rooftops (external surfaces) and attic walls (both external and internal surfaces) of the building. The tests were carried out for 24 wind directions with $15^{\circ}$ increment, as shown in Fig. 6.

Every measurement conducted in the wind tunnel results in 5,000 samples for each point (20 seconds measurement with sampling frequency of $250 \mathrm{~Hz}$ ). In wind tunnel conditions, 


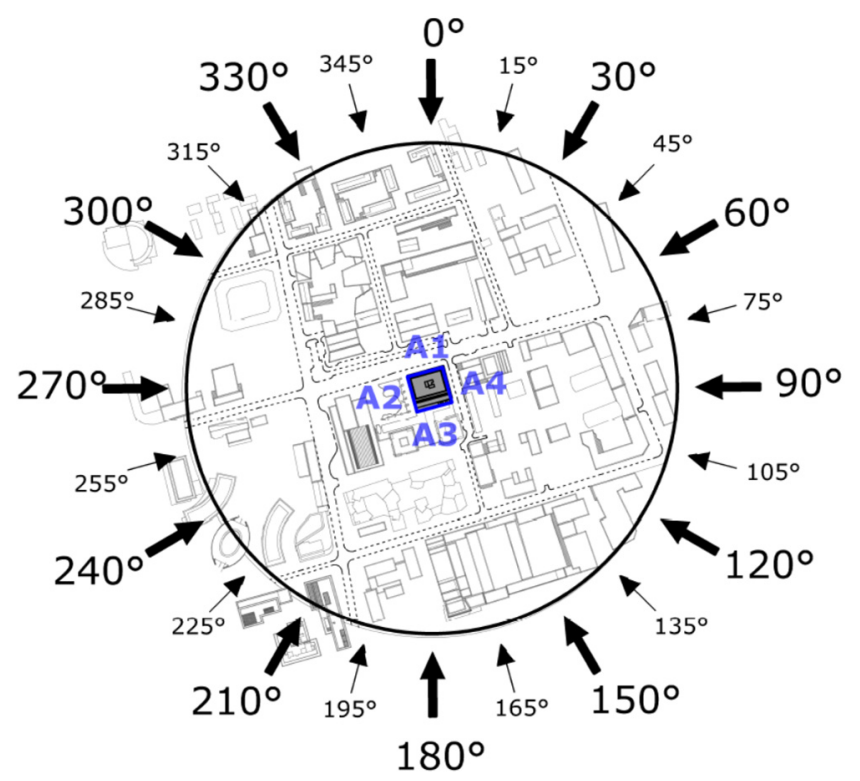

Figure 6: Wind directions investigated during wind tunnel tests.
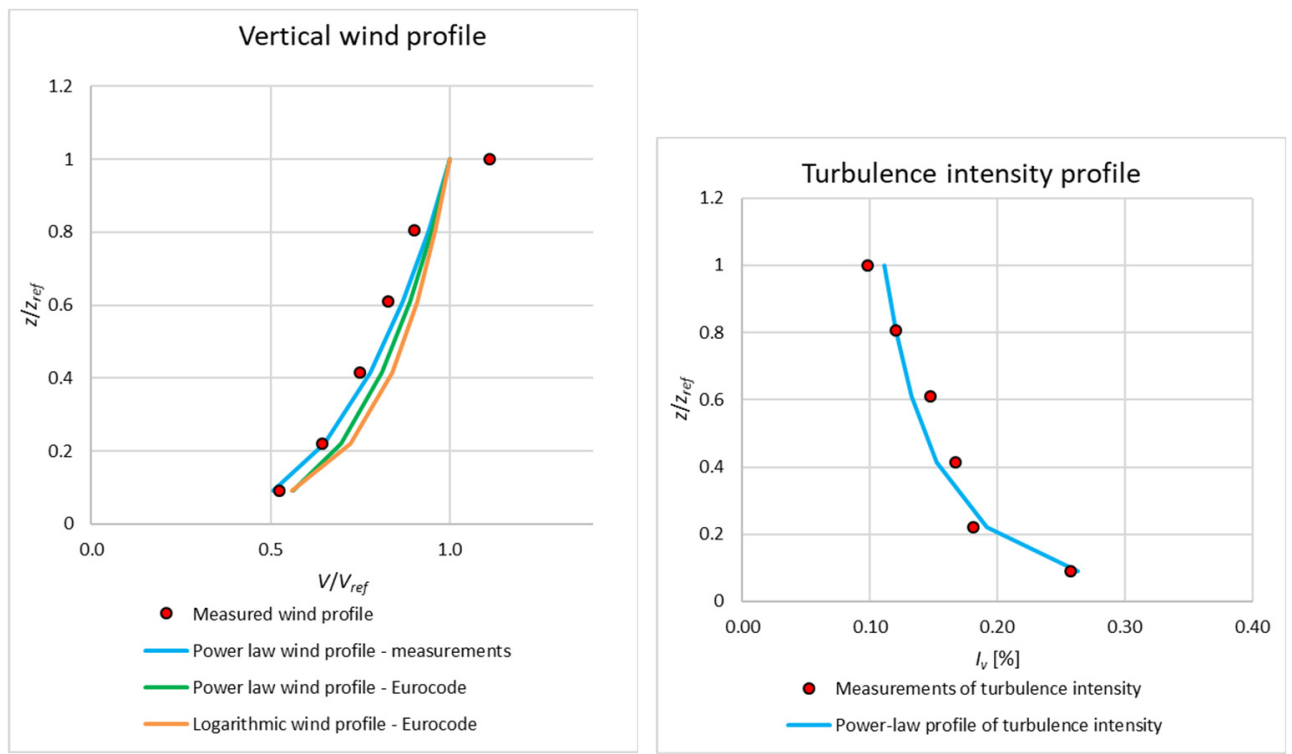

Figure 7: Vertical wind velocity and turbulence intensity profiles.

such measurement may correspond to mean 10-minute values of wind velocity provided in the building codes. The reference wind velocity (velocity at the reference height $z_{r e f}=57.5 \mathrm{~cm}$ in model scale) inside the wind tunnel during the tests was maintained at a level of about $12-12.5 \mathrm{~m} / \mathrm{s}$, as the most convenient range for the pressure scanners used. Graphs 
of vertical wind velocity and turbulence intensity profiles have been added to the manuscript in Fig. 7. A total of 383 measuring points were marked on the building (points 1-172 and 178-388 - points 173-177 where located on the bottom podium façade, which is directly adjacent to the neighbouring building, thus no measurements could be conducted there).

During the tests, three different values of pressure coefficient are determined at each measuring point and for each wind direction: mean pressure coefficient, maximal pressure coefficient and minimal pressure coefficient.

Extreme values are determined as $99 \%$ quantile (i.e. rejection of $1 \%$ peak values and then taking the maximum instantaneous wind pressure coefficient) from the dimensionless external wind pressure coefficients. The provision of maximal value quantile (usually $98 \%-$ $99 \%$ ) is a result of the necessity to eliminate errors at the stage of measurement signal processing. These errors result for example from the influence of wind tunnel fan large inverter or length of the tubes between pressure taps and pressure scanners. The decision on the exact value of this quantile is based on the experience of the authors in signal processing and is a direct consequence of the degree of these errors.

Mean values of pressure coefficient are mostly useful for the study of the general pressure distribution on the walls and roof. However, they do not consider the peak value of wind pressure, described in EN 1991-1-4, therefore their values are significantly lower than the characteristic wind pressures used for structural design and should not be used for this purpose.

The next step was dividing the walls and roof of the building to larger areas (in this case a total of 144 areas), where the pressure coefficients values will be spatially averaged between a number of measuring points. This is performed at each point in time series. The next step is performing time averaging and finding the extreme values for each of the areas. The results obtained from this method may be considered as characteristic peak values of wind pressure for each wind direction, at each of the areas. These results may be applied in the design process for global wind action analysis of the structure and should be considered individually for each wind angle of attack. The algorithm of this procedure is presented in Fig. 8.

Dividing the surfaces for measuring areas was established on the basis of measured wind velocity pressure values and the general principles of pressure distribution on flat surfaces. Therefore, points which are in the corners of each wall, which are especially sensitive to wind

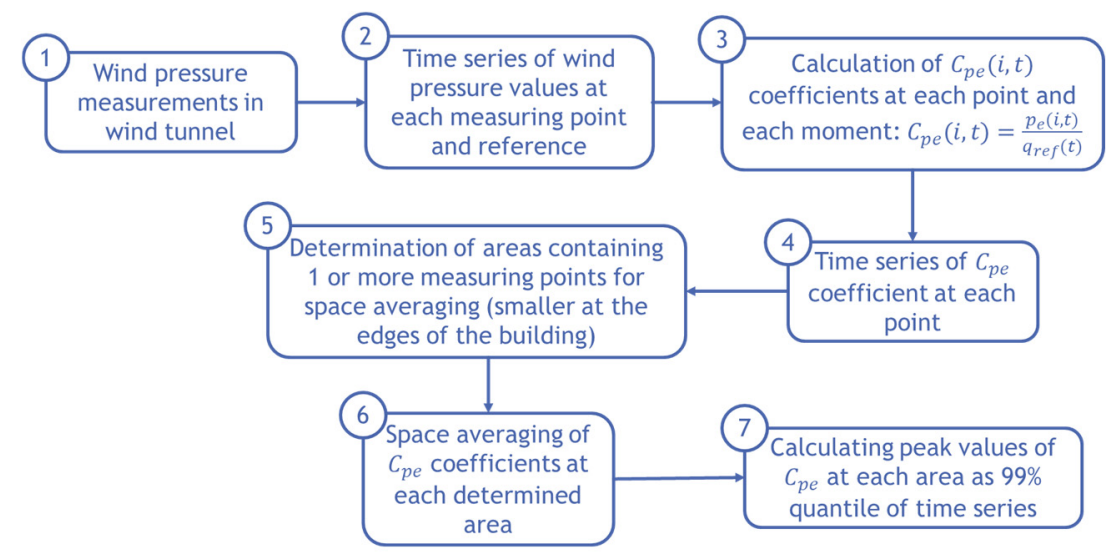

Figure 8: Algorithm of the proposed approach. 
effects, are located in their own, single areas. Similarly, points on the edges are grouped together without any points that are in the central part of a surface.

An example of practical use of the proposed approach for smoothening the effects of local, small scale gusts and the differences between this approach and simple time averaging are shown in Fig. 9 and Table 1.

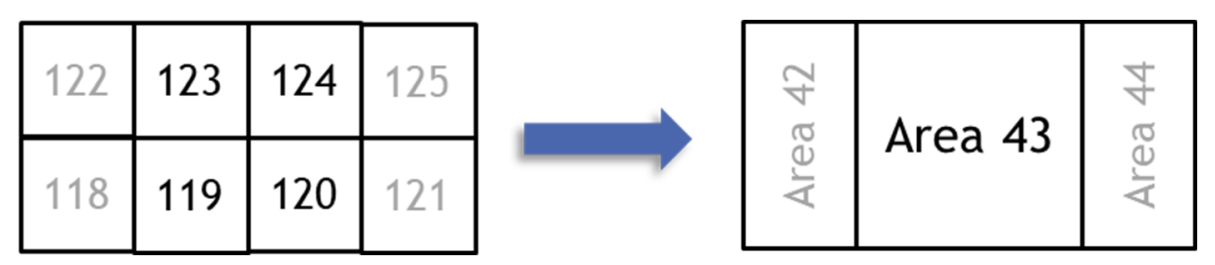

Figure 9: Example of determining measurement areas on external surfaces of the building.

Table 1: Influence of the proposed approach for peak values of pressure coefficients.

\begin{tabular}{|l|c|}
\hline Peak values (99\% quantile) of pressure coefficient \\
\hline Point number/area & Pressure coefficient value (-) \\
\hline Point 119 & -1.985 \\
\hline Point 120 & -1.952 \\
\hline Point 123 & -2.013 \\
\hline Point 124 & -1.886 \\
\hline Mean from points 119, 120, 123, 124 & -1.959 \\
\hline Area 43 & -1.896 \\
\hline
\end{tabular}

The last group of results obtained are maximal positive values (pressures) and minimal negative values (suctions) of wind pressures, which are collected for each point and from every wind direction. These values correspond to local values of external wind pressures $\left(C_{p e, 1}\right)$ and are useful for designing cladding elements (panels and covers) or other smaller elements.

\section{TEST RESULTS}

Exemplary results of global characteristic wind pressures, calculated on the basis of the proposed method, are shown in Fig. 10. Global horizontal aerodynamic forces and aerodynamic torsional moment acting at the level of the base of the building are presented in Table 2 and Fig. 11. The building's local aerodynamic coordinate system is shown in Fig. 12.

\section{CONCLUSIONS}

A detailed analysis of wind pressure coefficients distribution for each angle of attack allowed for a validation of the conducted tests. High-rise, dense development around the subject building causes strong aerodynamic interference, which has its influence on the pressure distribution on building's façades. Such effects might either be beneficial for the structure (shielding larger portions of the building at certain wind directions) or adverse (large scale vortex shedding or local flow restrictions, which may cause the amplification of wind velocity). 

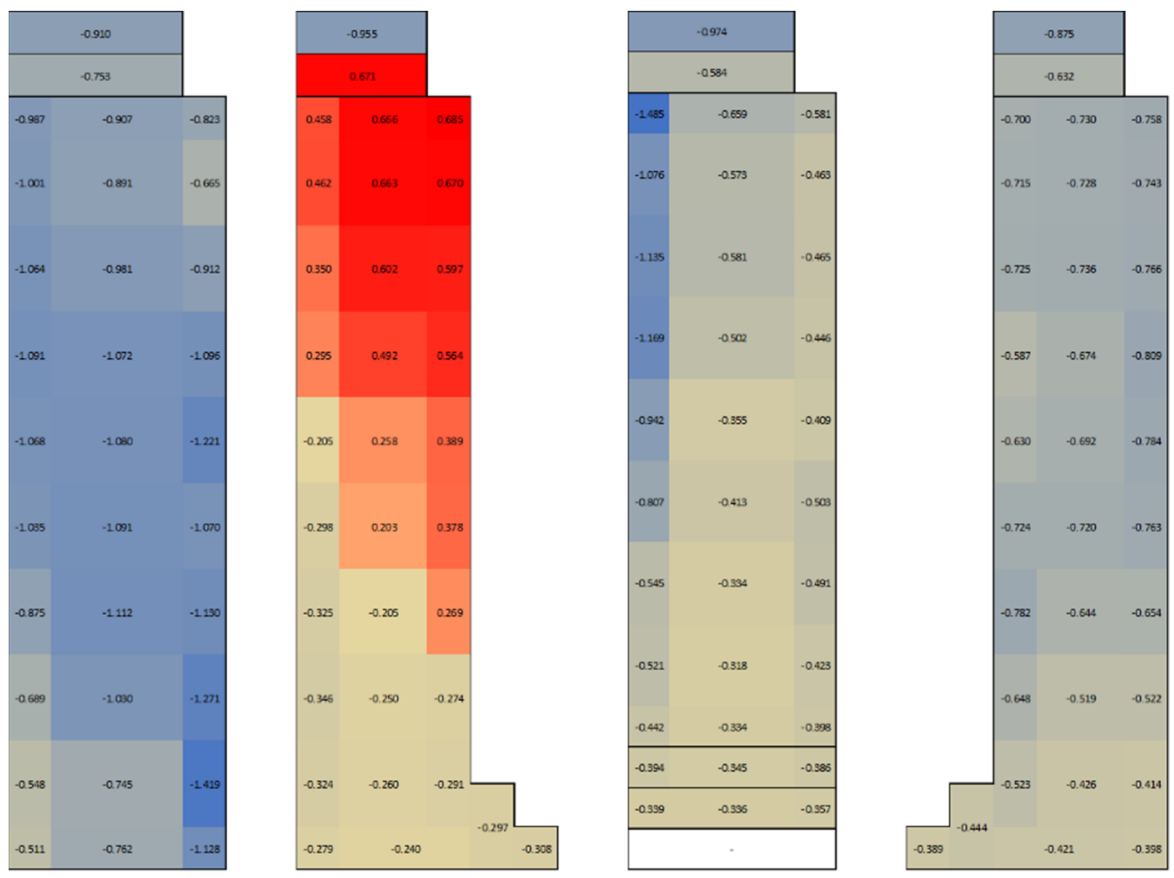

(a)
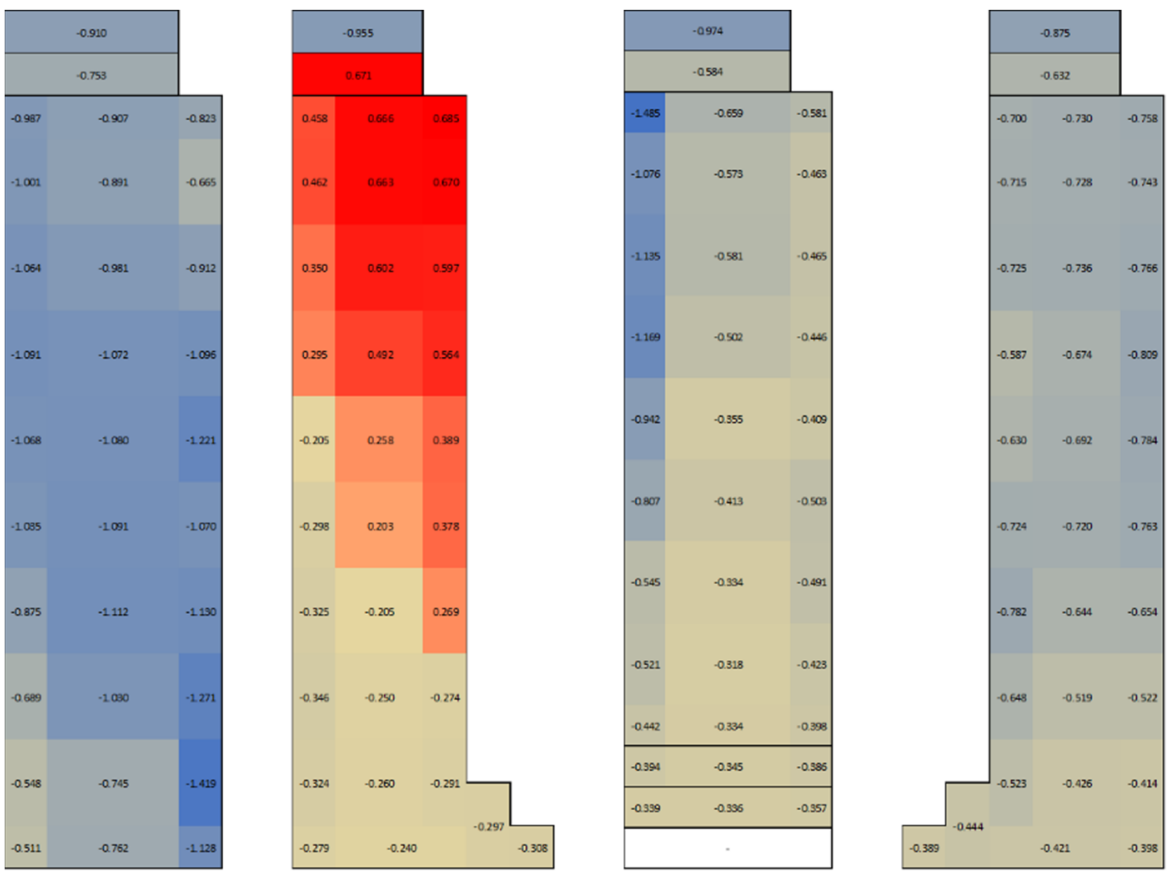

(b)

Figure 10: Exemplary results of global characteristic wind pressures $(\mathrm{kPa})$. (a) Wind angle of attack $240^{\circ}$; and (b) Wind angle of attack $330^{\circ}$. 
Table 2: Characteristic values of global aerodynamic forces on the base of the building.

\begin{tabular}{|c|c|c|c|}
\hline Wind direction & $F_{x}(\mathrm{kN})$ & $F_{y}(\mathrm{kN})$ & $M_{z}(\mathrm{kNm})$ \\
\hline $0^{\circ}$ & 154.7 & $-6,204.8$ & $-11,017.6$ \\
\hline $15^{\circ}$ & $-1,206.7$ & $-6,635.9$ & $-19,068.2$ \\
\hline $30^{\circ}$ & $-3,229.5$ & $-5,928.4$ & $-8,847.7$ \\
\hline $45^{\circ}$ & $-3,888.7$ & $-3,766.5$ & $3,196.0$ \\
\hline $60^{\circ}$ & $-4,015.1$ & $-2,159.9$ & $7,785.2$ \\
\hline $75^{\circ}$ & $-3,305.4$ & -39.8 & -153.5 \\
\hline $90^{\circ}$ & $-3,504.2$ & $3,139.9$ & $-2,660.3$ \\
\hline $105^{\circ}$ & $-4,578.8$ & $3,843.6$ & $-3,385.5$ \\
\hline $120^{\circ}$ & $-3,594.7$ & $4,398.8$ & 317.6 \\
\hline $135^{\circ}$ & $-1,730.8$ & $5,270.0$ & $9,554.8$ \\
\hline $150^{\circ}$ & -124.0 & $5,735.3$ & $6,141.0$ \\
\hline $165^{\circ}$ & 237.7 & $6,928.5$ & $1,168.2$ \\
\hline $180^{\circ}$ & -2.8 & $6,234.0$ & $-7,373.8$ \\
\hline $195^{\circ}$ & 960.6 & $6,050.4$ & $-18,070.7$ \\
\hline $210^{\circ}$ & $2,919.0$ & $4,020.3$ & $-7,432.8$ \\
\hline $225^{\circ}$ & $4,166.6$ & $3,215.0$ & $6,169.9$ \\
\hline $240^{\circ}$ & $4,369.4$ & $3,334.0$ & $10,127.9$ \\
\hline $255^{\circ}$ & $3,475.2$ & 993.7 & -244.1 \\
\hline $270^{\circ}$ & $3,646.6$ & $-1,754.5$ & $-8,772.4$ \\
\hline $285^{\circ}$ & $4,012.1$ & $-3,130.5$ & $-11,392.6$ \\
\hline $300^{\circ}$ & $2,782.3$ & $-5,094.4$ & $10,910.0$ \\
\hline $315^{\circ}$ & $2,362.4$ & $-6,444.0$ & $21,041.2$ \\
\hline $330^{\circ}$ & 263.6 & $-7,083.0$ & $9,376.4$ \\
\hline $345^{\circ}$ & 595.2 & $-6,955.4$ & 149.2 \\
\hline
\end{tabular}

Maximal characteristic local wind pressures reach up to $0.7-0.8 \mathrm{kPa}$ and can be observed mostly in the upper part of the building. This is present on each of the façades. Largest characteristic values of wind pressures are respectively $0.773 \mathrm{kPa}$ on A1 (point 91), $0.811 \mathrm{kPa}$ on A2 (point 172), $0.854 \mathrm{kPa}$ on A3 (point 262) and $0.804 \mathrm{kPa}$ on A4 (point 347).

Largest (absolute) values of local suctions are usually noticed around the edges of the building in middle and upper parts along the height. These values are significantly lower on the lower part of the building. Largest values on each of the façades are respectively: $-1.920 \mathrm{kPa}$ on A1 (point 60), $-1.824 \mathrm{kPa}$ on A2 (point 169), $-2.104 \mathrm{kPa}$ on A3 (point 223) and $-1.572 \mathrm{kPa}$ on A4 (point 311 ).

Both on rooftop and the terraces, absolute values of negative wind pressure (suction) are significantly larger than values of positive wind pressure - they reach values of up to $-1.362 \mathrm{kPa}$ on the roof (point 367), $-1.069 \mathrm{kPa}$ on terrace 1 (point 378) and $-1.396 \mathrm{kPa}$ on terrace 2 (point 381 ).

Largest values of aerodynamic force $\left(F_{x} \mathrm{i} F_{y}\right)$ are observed, as expected, at wind directions which are perpendicular (or almost perpendicular) to one of the façades. Largest absolute values of global horizontal force $F_{x}$ can be observed for wind directions $105^{\circ}(-4,578.8 \mathrm{kN})$ and $240^{\circ}(4,369.4 \mathrm{kN})$. Similarly, largest absolute values of global horizontal force $F_{x}$ are present for wind directions $165^{\circ}\left(6,928,5 \mathrm{kN}\right.$ ) and $330^{\circ}$ and $345^{\circ}$ (respectively: $-7,083.0 \mathrm{kN}$, $-6,955.4 \mathrm{kN})$. Torsional moment $M_{z}$ reaches its highest absolute value at wind directions $15^{\circ}$ 


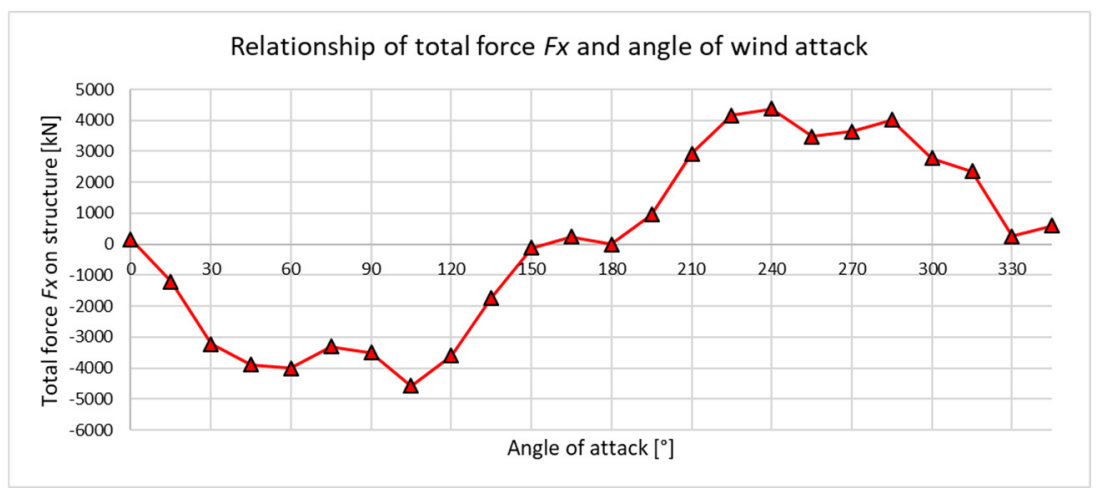

(a)

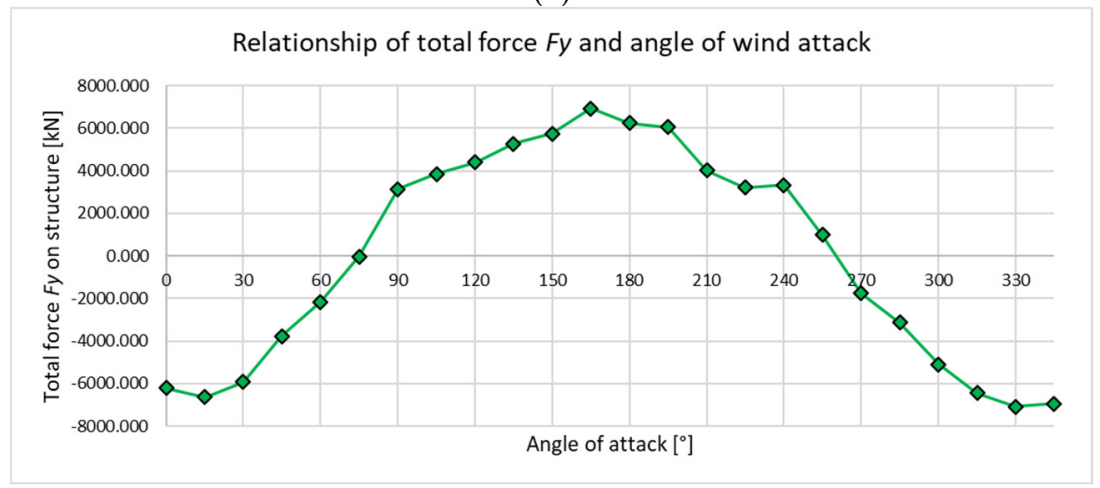

(b)

Figure 11: Relationship of total horizontal force on wind angle of attack (a) $F_{x}$ (b) $F_{y}$.

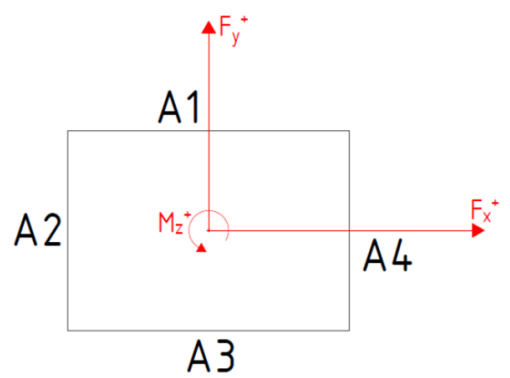

Figure 12: Local aerodynamic coordinate system of the building.

$(-19,068.2 \mathrm{kNm})$ and $315^{\circ}(21,041.2 \mathrm{kNm})$. Relations between wind direction and global forces shown in graphs in Fig. 12 allow for easy identification of the wind directions where aerodynamic forces acting on the structure reach their highest values.

The measurement error in such tests has to be estimated basing on the following variables: precision of the measuring equipment (in the case of pressure sensors, it is at the level of $0.03 \%$ ); model precision (buildings shapes, accuracy on wind directions); blockage effect 
(mostly significant on the edges of the whole model - in case where the building is in the centre, this effect does not affect results significantly); the precision level of re-creating the turbulence. On the summary of all these factors, the measuring error can be estimated at below $10 \%$, which is sufficient for engineering applications.

\section{ACKNOWLEDGEMENTS}

Wind tunnel model tests presented here are a result of commissioned work conducted for Kuryłowicz \& Associates - consulting architects and in cooperation with consulting engineers - WSP Poland.

\section{REFERENCES}

[1] Flaga, A. et al., Wind tunnel model tests of high-rise building Skyreach located at Grzybowska 59 in Warsaw, Kraków, 2020. (In Polish.)

[2] Flaga, A., Pistol, A., Krajewski, P. \& Flaga, Ł., Cold regions science and technology aerodynamic and aeroelastic wind tunnel model tests of overhead power lines in triangular configuration under different icing conditions. Cold Reg. Sci. Technol., 170, 102919, 2020. https://doi.org/10.1016/j.coldregions.2019.102919.

[3] Niemann, H.J., Dynamic response of contilevered structures to wind turbulence. Proc. Eur. Conf. Struct. Dyn. EURODYN'90, Bochum, Germany, pp. 1123-1131, 1991.

[4] Cook, N.J., The Designer's Guide to Wind Loading of Building Structures. Part II. Static Structures, 1990.

[5] EN 1991-1-4, Actions on Structures. General Actions. Wind Actions, 2, 2011.

[6] Flaga, A., Quasi-steady models of wind load on slender structures. Part I. Case of a motionless structure. Arch. Civ. Eng. XL, pp. 3-28, 1994.

[7] Flaga, A., Quasi-steady models of wind load on slender structures. Part II. Case of a moving structure. Arch. Civ. Eng. XL, pp. 29-41, 1994.

[8] Flaga, A., Quasi-steady models of wind load on slender structures. Part III. Aplications of quasi-steady theory in aerodynamics of slender structures. Arch. Civ. Eng. XLI, pp. 343-376, 1995.

[9] Flaga, A., Wind engineering: Fundamentals and applications, Arkady: Warsaw, Poland, 2008. (In Polish.) 